\title{
THE CAUCHY PROBLEM FOR THE SCHRÖDINGER EQUATION IN DIMENSION THREE WITH CONCENTRATED NONLINEARITY
}

\section{LE PROBLÈME DE CAUCHY POUR UNE ÉQUATION DE SCHRÖDINGER EN TROIS DIMENSIONS AVEC UNE NONLINÉARITÉ CONCENTRÉE}

\author{
Riccardo ADAMI ${ }^{\mathrm{a}, *}$, Gianfausto DELL'ANTONIO ${ }^{\mathrm{b}, \mathrm{c}}$, Rodolfo FIGARI $^{\mathrm{d}}$, \\ Alessandro TETA ${ }^{\mathrm{e}}$ \\ a Département de mathématiques et applications, École Normale Supérieure, Paris, France \\ b Dipartimento di Matematica, Università di Roma "La Sapienza", Italy \\ ${ }^{\mathrm{c}}$ Laboratorio Interdisciplinare SISSA-ISAS, Trieste, Italy \\ d Dipartimento di Scienze Fisiche, Università di Napoli "Federico II", Italy \\ e Dipartimento di Matematica Pura e Applicata, Università di L'Aquila, Italy
}

Received 11 November 2001

ABSTRACT. - We consider the Schrödinger equation in $\mathbb{R}^{3}$ with nonlinearity concentrated in a finite set of points. We formulate the problem in the space of finite energy $V$, which is strictly larger than the standard $H^{1}$-space due to the specific singularity exhibited by the solutions. We prove local existence and, for a repulsive or weakly attractive nonlinearity, also global existence of the solutions.

(C) 2003 L'Association Publications de l'Institut Henri Poincaré. Published by Elsevier B.V. All rights reserved

Keywords: Nonlinear Schrödinger Equation (NLSE); Point interactions; Nonlinear Dirac Delta potentials; Existence and uniqueness in energy space

RÉSUMÉ. - On considère l'équation de Schrödinger avec une nonlinéarité concentrée en un nombre fini de points. On formule le problème dans l'espace $V$ d'énergie finie, qui contient strictement l'espace standard $H^{1}$, à cause de la singularité spécifique des solutions. On prouve des résultats d'existence locale et même, pour une nonlinéarité répulsive ou faiblement attractive, l'existence globale des solutions.

(C) 2003 L'Association Publications de l'Institut Henri Poincaré. Published by Elsevier B.V. All rights reserved

\footnotetext{
* Corresponding author.

E-mail addresses: riccardo.adami@ens.fr (R. Adami), gianfa@sissa.it (G. Dell’Antonio), figari@na.infn.it (R. Figari), teta@univaq.it (A. Teta).
} 


\section{Introduction}

In this note we introduce a model of nonlinear Schrödinger equation in $\mathbb{R}^{3}$ with a nonlinearity concentrated in a finite number of points. The corresponding case in dimension one was studied in [1,2]. The three-dimensional case exhibits a more singular character. As a consequence, the formulation of the problem and the techniques used are not a straightforward generalization of the one-dimensional case.

In order to introduce the model it is convenient to start with the linear case. It is well known [4] that, for given $\alpha_{1}, \ldots, \alpha_{n}$ and $\mathbf{y}_{1}, \ldots, \mathbf{y}_{n}$, with $\alpha_{j} \in \mathbb{R}, \mathbf{y}_{j} \in \mathbb{R}^{3}, j=1, \ldots n$, one can define the Schrödinger operator in $\mathbb{R}^{3}$ with local point interactions at $\mathbf{y}_{1}, \ldots, \mathbf{y}_{n}$ with strength $\alpha_{1}, \ldots, \alpha_{n}$. Such operator, denoted by $H_{\alpha}$, where $\alpha=\left(\alpha_{1}, \ldots, \alpha_{n}\right)$, is selfadjoint and bounded from below in $L^{2}\left(\mathbb{R}^{3}\right)$. The domain and action are respectively given by

$$
\begin{gathered}
D\left(H_{\alpha}\right)=\left\{u \in L^{2}\left(\mathbb{R}^{3}\right) \mid u=\phi+\sum_{j=1}^{n} q_{j} G_{0}\left(\cdot-\mathbf{y}_{j}\right), \phi \in H_{\mathrm{loc}}^{2}\left(\mathbb{R}^{3}\right),\right. \\
\nabla \phi \in L^{2}\left(\mathbb{R}^{3}\right), \Delta \phi \in L^{2}\left(\mathbb{R}^{3}\right),\left(q_{1}, \ldots, q_{n}\right) \in \mathbb{C}^{n}, \\
\left.\lim _{\mathbf{x} \rightarrow \mathbf{y}_{j}}\left(u(\mathbf{x})-q_{j} G_{0}\left(\mathbf{x}-\mathbf{y}_{j}\right)\right)=\alpha_{j} q_{j}, j=1, \ldots, n\right\}, \\
H_{\alpha} u=-\Delta \phi,
\end{gathered}
$$

where $G_{\lambda}$ denotes the Green's function

$$
G_{\lambda}\left(\mathbf{x}-\mathbf{x}^{\prime}\right)=(-\Delta+\lambda)^{-1}\left(\mathbf{x}-\mathbf{x}^{\prime}\right)=\frac{\mathrm{e}^{-\sqrt{\lambda}\left|\mathbf{x}-\mathbf{x}^{\prime}\right|}}{4 \pi\left|\mathbf{x}-\mathbf{x}^{\prime}\right|}, \quad \lambda \geqslant 0 .
$$

Roughly speaking, the operator $H_{\alpha}$ should be understood as the laplacian with a generalized boundary condition at $\mathbf{y}_{1} \ldots \mathbf{y}_{n}$ expressed by the limit relations in (1.1).

Note that in the literature the generalized boundary condition is often written in the following equivalent form

$$
\left.\frac{\partial}{\partial r_{j}}\left(r_{j} u(\mathbf{x})\right)\right|_{r_{j}=0}=\left.\alpha_{j}\left(r_{j} u(\mathbf{x})\right)\right|_{r_{j}=0}, \quad r_{j}=\left|\mathbf{x}-\mathbf{y}_{j}\right| .
$$

It is clear from (1.4) that the boundary condition is local in the sense that it couples the (renormalized) value of the function and of its radial derivative at the same point $\mathbf{y}_{j}$.

One can also introduce the quadratic form associated to the operator $H_{\alpha}$

$$
F_{\alpha}(u)=\int_{\mathbb{R}^{3}} d \mathbf{x}|\nabla \phi(\mathbf{x})|^{2}+\sum_{j=1}^{n} \alpha_{j}\left|q_{j}\right|^{2}-\sum_{j \neq k} \frac{\overline{q_{j}} q_{k}}{4 \pi\left|\mathbf{y}_{j}-\mathbf{y}_{k}\right|}
$$

defined on the following form domain or space of finite energy

$$
V=\left\{u \in L^{2}\left(\mathbb{R}^{3}\right) \mid u=\phi+\sum_{j=1}^{n} q_{j} G_{0}\left(\cdot-\mathbf{y}_{j}\right), \phi \in H_{\mathrm{loc}}^{1}\left(\mathbb{R}^{3}\right),\right.
$$




$$
\left.\nabla \phi \in L^{2}\left(\mathbb{R}^{3}\right), q_{1}, \ldots, q_{n} \in \mathbb{C}\right\}
$$

which can also be written in the following equivalent form (for $\lambda>0$ )

$$
V=\left\{u \in L^{2}\left(\mathbb{R}^{3}\right) \mid u=\phi_{\lambda}+\sum_{j=1}^{n} q_{j} G_{\lambda}\left(\cdot-\mathbf{y}_{j}\right), \phi_{\lambda} \in H^{1}\left(\mathbb{R}^{3}\right), q_{1}, \ldots, q_{n} \in \mathbb{C}\right\}
$$

(see e.g. [14]).

Note that the space $V$ is strictly larger than $H^{1}\left(\mathbb{R}^{3}\right)$, so $H_{\alpha}$ cannot be defined as a small perturbation of the laplacian in the sense of the quadratic forms. We stress that this is the main difference with respect to the one dimensional case, where the form domain of the operator is in fact $H^{1}(\mathbb{R})$.

We shall consider the case where the $\alpha$ 's depend on time. Let $\alpha(t)$ be a sufficiently smooth function of $t$ in $\mathbb{R}^{n}$ and let $H_{\alpha(t)}$ be the corresponding family of operators. Notice that $D\left(H_{\alpha(t)}\right)$ is varying with time whereas the form domain remains the same for all $t$.

The linear evolution problem associated to the time dependent generator $H_{\alpha(t)}$ is

$$
i \frac{\partial}{\partial t} \psi(t)=H_{\alpha(t)} \psi(t), \quad \psi(0)=\psi_{0}
$$

Following [13], the strong solution of (1.8) can be written in the form

$$
\psi(t, \mathbf{x})=\left(U(t) \psi_{0}\right)(\mathbf{x})+i \sum_{j=1}^{n} \int_{0}^{t} d s U\left(t-s, \mathbf{x}-\mathbf{y}_{j}\right) q_{j}(s)
$$

where $U(t)$ is the free unitary group defined by the integral kernel

$$
U\left(t, \mathbf{x}-\mathbf{x}^{\prime}\right)=\frac{\mathrm{e}^{i \frac{\left|\mathbf{x}-\mathbf{x}^{\prime}\right|^{2}}{4 t}}}{(4 \pi i t)^{3 / 2}}
$$

and the functions $q_{j}(t)$ solve the system of linear Volterra integral equations

$$
\begin{aligned}
& q_{j}(t)+4 \sqrt{\pi i} \int_{0}^{t} d s \frac{\alpha_{j}(s) q_{j}(s)}{\sqrt{t-s}}-\sum_{l=1, l \neq j}^{n} \frac{\sqrt{i}}{\sqrt{\pi}\left|\mathbf{y}_{j}-\mathbf{y}_{l}\right|} \int_{0}^{t} d s \frac{\mathrm{e}^{i \frac{\left|\mathbf{y}_{j}-\mathbf{y}_{l}\right|^{2}}{4(t-s)}}}{\sqrt{t-s}} q_{l}(s) \\
& \quad=4 \sqrt{\pi i} \int_{0}^{t} d s \frac{\left[U(s) \psi_{0}\right]\left(\mathbf{y}_{j}\right)}{\sqrt{t-s}} .
\end{aligned}
$$

Our nonlinear evolution problem in its integral form is then defined by considering the strength of the interaction $\alpha(t)$ as a function of the solution itself. Note that this corresponds to impose a nonlinear boundary condition at $\mathbf{y}_{j}$ (cfr. the limit in (1.1)). We fix

$$
\alpha_{j}(z)=\gamma_{j} z^{\sigma_{j}}, \quad z=\left|q_{j}(t)\right|^{2}, \quad \gamma_{j} \in \mathbb{R}, \sigma_{j} \in \mathbb{R}^{+} .
$$


With this choice the solution of the nonlinear evolution problem is given by (1.9) where the $q_{j}(t)$ 's solve the system of nonlinear Volterra equations

$$
\begin{aligned}
& q_{j}(t)+4 \sqrt{\pi i} \gamma_{j} \int_{0}^{t} d s \frac{\left|q_{j}(s)\right|^{2 \sigma_{j}} q_{j}(s)}{\sqrt{t-s}}-\sum_{l=1, l \neq j}^{n} \frac{\sqrt{i}}{\sqrt{\pi}\left|\mathbf{y}_{j}-\mathbf{y}_{l}\right|} \int_{0}^{t} d s \frac{e^{i \frac{\left|\mathbf{y}_{j}-\mathbf{y}_{l}\right|^{2}}{4(t-s)}}}{\sqrt{t-s}} q_{l}(s) \\
& =4 \sqrt{\pi i} \int_{0}^{t} d s \frac{\left[U(s) \psi_{0}\right]\left(\mathbf{y}_{j}\right)}{\sqrt{t-s}} .
\end{aligned}
$$

We will prove that (1.9), (1.13) define a flow on constant energy surfaces in $V$, even if, for technical reasons, in a first stage we will consider slightly more regular initial data.

We emphasize that the evolution problem (1.9), (1.13) cannot be analyzed following the usual techniques of the standard nonlinear Schrödinger equation $[5,6,8,11,15]$, since the space $V$ is strictly larger than $H^{1}\left(\mathbb{R}^{3}\right)$. In particular, this fact prevents a direct application of Sobolev inequalities, which are the typical tools for proving existence theorems.

We collect here some notation which will be used in the sequel.

We shall denote by $r$ and $k$ the modulus of the three-dimensional vectors $\mathbf{x}$ and $\mathbf{k}$ respectively.

We shall indicate with the same symbol the same function although expressed in different coordinates: $\psi(\mathbf{x})$ and $\psi(r, \vartheta, \varphi)$ will be the same if the vector $\mathbf{x}$ is represented in polar coordinates by $(r, \vartheta, \varphi)$.

The Fourier transform with respect to the space variables will be denoted by $\hat{\psi}$, i.e.

$$
\begin{gathered}
\hat{\psi}(\mathbf{k})=\int_{\mathbb{R}^{3}} d \mathbf{x} \mathrm{e}^{i \mathbf{k} \cdot \mathbf{x}} \psi(\mathbf{x}), \\
\psi(\mathbf{x})=(2 \pi)^{-3} \int_{\mathbb{R}^{3}} d \mathbf{k} \mathrm{e}^{-i \mathbf{k} \cdot \mathbf{x}} \hat{\psi}(\mathbf{k}) .
\end{gathered}
$$

The symbol $\mathcal{F} f$ will denote the Fourier transform of the function $f$ with respect to the time variable

$$
\begin{gathered}
(\mathcal{F} f)(\omega)=\int_{\mathbb{R}} d t \mathrm{e}^{i \omega t} f(t), \\
f(t)=(2 \pi)^{-1} \int_{\mathbb{R}} d \omega \mathrm{e}^{-i \omega t}(\mathcal{F} f)(\omega) .
\end{gathered}
$$

Given $T \in[0,+\infty]$ and $f:(0, T) \rightarrow \mathbb{C}$, we define the function $\tilde{f}^{a, b}: \mathbb{R} \rightarrow \mathbb{C}, 0 \leqslant a<$ $b \leqslant T$

$$
\tilde{f}^{a, b}(\tau)=f(\tau) \quad \text { if } \tau \in[a, b], \quad \tilde{f}^{a, b}(\tau)=0 \quad \text { if } \tau \notin[a, b] .
$$

In what follows we will often use fractional Sobolev spaces. We recall some basic definitions [3]. 
DeFINITION 1. - For $0<s<1,1 \leqslant p<+\infty, a, b \in[-\infty,+\infty]$, $a<b$, we denote by $W^{s, p}(a, b)$ the subspace of functions $f$ in $L^{p}(a, b)$ such that

$$
\int_{[a, b]^{2}} d u d v \frac{|f(u)-f(v)|^{p}}{|u-v|^{1+s p}}<\infty .
$$

The spaces $W^{s, p}(a, b)$ are Banach spaces endowed with the norm:

$$
\|f\|_{W^{s, p}(a, b)}^{p}=\|f\|_{L^{p}(a, b)}^{p}+\int_{[a, b]^{2}} d u d v \frac{|f(u)-f(v)|^{p}}{|u-v|^{1+s p}} .
$$

The spaces $W^{s, 2}(a, b)$ are usually denoted by $H^{s}(a, b)$.

In the case $a=-\infty, b=+\infty$ one can equivalently define $H^{s}(\mathbb{R})$ requiring $f \in$ $L^{2}(\mathbb{R})$ and

$$
\left.\left.\int_{\mathbb{R}} d \omega|| \omega\right|^{s}(\mathcal{F} f)(\omega)\right|^{2}<+\infty
$$

The corresponding norm is defined as follows

$$
\|f\|_{H^{s}(\mathbb{R})}^{2}=\|f\|_{L^{2}(\mathbb{R})}^{2}+\left.\left.\int_{\mathbb{R}} d \omega|| \omega\right|^{s}(\mathcal{F} f)(\omega)\right|^{2} .
$$

The remaining part of the paper is organized as follows.

In Section 2 we prove the local existence of the solution of (1.9), (1.13) in $V$ for a restricted class of initial data.

In Section 3 we show that the solution satisfies the boundary condition given in (1.1) at any time of existence.

In Section 4 we extend the local existence result to allow the initial datum to be an arbitrary element of $V$.

In Section 5 we prove the conservation laws of the $L^{2}$-norm and the energy of the solution.

In Section 6 we use the conservation laws to obtain a global existence theorem in the repulsive and in the weakly attractive case.

\section{Local existence for a restricted class of initial data}

We shall consider the local (in time) existence problem in the space $V$ for an initial datum $\psi_{0}=\phi_{0 \lambda}+\sum_{j=1}^{n} q_{j 0} G_{\lambda}\left(\cdot-\mathbf{y}_{j}\right), \phi_{0 \lambda} \in H^{2}\left(\mathbb{R}^{3}\right)$.

We start recalling some technical lemmas proven in [2].

Lemma 2. - Let $f \in H^{\beta}(\mathbb{R})$ with support in $[0, T], T<\infty, \beta \geqslant 0$, and let

$$
F(t)=\int_{0}^{t} d s \frac{f(s)}{\sqrt{t-s}}
$$


Then $F$ is an element of $H_{\mathrm{loc}}^{\beta+1 / 2}(\mathbb{R})$.

LEMma 3. - Given $\rho \in[0,1 / 2)$ and $T>0$ consider $f \in H^{\rho}(0, T) \cap C^{0}([0, T])$. Then $\tilde{f}^{a, b} \in H^{\rho}(\mathbb{R})$ for any $a, b \in[0, T]$.

Now we can prove the main result of this section:

THEOREM 4. - Let $\psi_{0}=\phi_{0 \lambda}+\sum_{j=1}^{n} q_{j 0} G_{\lambda}\left(\cdot-\mathbf{y}_{j}\right), \phi_{0 \lambda} \in H^{2}\left(\mathbb{R}^{3}\right)$. Then $\exists T>0$ such that for any $t \in[0, T]$ problem (1.9), (1.13) has a unique solution $\psi(t) \in V$.

Proof. - The proof proceeds in several steps. In the first step we compute the Sobolev degree of the inhomogeneous term in Eq. (1.13); in the second step we prove the existence and uniqueness result and study the regularity of the solution of (1.13); in the third step we show how this regularity transfers to the function $\phi_{\lambda}(t)$, via (1.9), and conclude that the regular part of the solution actually lies in $H^{1}\left(\mathbb{R}^{3}\right)$ at any time of existence.

Step 1 . First we introduce the mean value over the unit sphere, both in position space and in momentum space, as a continuous map from $L^{2}\left(\mathbb{R}^{3}\right)$ to $L^{2}\left(\mathbb{R}^{+}, r^{2} d r\right)$

$$
\begin{aligned}
& \psi^{m}(r)=\frac{1}{4 \pi} \int_{0}^{2 \pi} d \varphi \int_{0}^{\pi} \psi(r, \theta, \varphi) \sin \theta d \theta, \\
& (\hat{\psi})^{m}(k)=\frac{1}{4 \pi} \int_{0}^{2 \pi} d \zeta \int_{0}^{\pi} \hat{\psi}(k, \eta, \zeta) \sin \eta d \eta .
\end{aligned}
$$

We recall that $(\hat{\psi})^{m}(k)=\widehat{\psi^{m}}(k)$.

Moreover, we denote by $T_{j}$ the translation by the vector $\mathbf{y}_{j}$

$$
\left(T_{j} f\right)(\mathbf{x})=f\left(\mathbf{x}-\mathbf{y}_{j}\right) .
$$

For any $\mathbf{y}_{j}$ we have

$$
\begin{aligned}
{\left[U(t) \phi_{0 \lambda}\right]\left(\mathbf{y}_{j}\right) } & \left.=\frac{1}{2 \pi^{2}} \int_{0}^{\infty} d k k^{2} \mathrm{e}^{-i k^{2} t} \widehat{T_{j}^{-1} \phi_{0 \lambda}}\right)^{m}(k) \\
& \left.=\frac{1}{4 \pi^{2}} \int_{0}^{\infty} d \omega \sqrt{\omega} \mathrm{e}^{-i \omega t} \widehat{\left(T_{j}^{-1} \phi_{0 \lambda}\right.}\right)^{m}(\sqrt{\omega}),
\end{aligned}
$$

then

$$
\left[\mathcal{F}\left(U(\cdot) \phi_{0 \lambda}\right)\left(\mathbf{y}_{j}\right)\right](\omega)=\frac{1}{2 \pi} \theta(\omega) \sqrt{\omega}\left(\widehat{T_{j}^{-1} \phi_{0 \lambda}}\right)^{m}(\sqrt{\omega}),
$$

where $\theta$ denotes the Heaviside step function.

It is now easy to evaluate the Sobolev degree of $\left(U(t) \phi_{0 \lambda}\right)\left(\mathbf{y}_{j}\right)$ as a function of $t$. Indeed, from (2.6),

$$
\left.\left.\left.\int_{\mathbb{R}} d \omega|| \omega\right|^{\beta}\left(\mathcal{F}\left[\left(U(\cdot) \phi_{0 \lambda}\right)\left(\mathbf{y}_{j}\right)\right]\right)(\omega)\right|^{2}=\frac{1}{2 \pi^{2}} \int_{0}^{\infty} d k k^{4 \beta+3} \mid \widehat{\left(T_{j}^{-1} \phi_{0 \lambda}\right.}\right)\left.^{m}(k)\right|^{2} .
$$


Using the Schwarz inequality

$$
\left|\int_{0}^{2 \pi} d \zeta \int_{0}^{\pi} d \eta \sin \eta g(k, \eta, \zeta)\right|^{2} \leqslant 4 \pi \int_{0}^{2 \pi} d \zeta \int_{0}^{\pi} d \eta \sin \eta|g(k, \eta, \zeta)|^{2} \text { a.e. in } k
$$

one obtains

$$
\left.\left.\int_{\mathbb{R}} d \omega|| \omega\right|^{\beta}\left(\mathcal{F}\left[\left(U(\cdot) \phi_{0 \lambda}\right)\left(\mathbf{y}_{j}\right)\right]\right)(\omega)\right|^{2} \leqslant \frac{2}{\pi} \int_{\mathbb{R}^{3}} d \mathbf{k} k^{4 \beta+1}\left|\widehat{T_{j}^{-1} \phi_{0 \lambda}}(\mathbf{k})\right|^{2}
$$

which is finite if $-1 / 4 \leqslant \beta \leqslant 3 / 4$ and $\phi_{0 \lambda} \in H^{2}\left(\mathbb{R}^{3}\right)$. In particular this implies that $\left(U(t) \phi_{0 \lambda}\right)\left(\mathbf{y}_{j}\right) \in H^{3 / 4}(\mathbb{R})$.

For $k \neq j$, we consider

$$
\begin{aligned}
{\left[U(t) T_{j} G_{\lambda}\right]\left(\mathbf{y}_{k}\right) } & =\frac{1}{2 \pi^{2}\left|\mathbf{y}_{k}-\mathbf{y}_{j}\right|} \int_{0}^{\infty} d k \frac{k \sin \left(k\left|\mathbf{y}_{k}-\mathbf{y}_{j}\right|\right)}{k^{2}+\lambda} \mathrm{e}^{-i k^{2} t} \\
& =\frac{1}{4 \pi^{2}\left|\mathbf{y}_{k}-\mathbf{y}_{j}\right|} \int_{0}^{\infty} d \omega \mathrm{e}^{-i \omega t} \frac{\sin \left(\sqrt{\omega}\left|\mathbf{y}_{k}-\mathbf{y}_{j}\right|\right)}{\omega+\lambda}
\end{aligned}
$$

From (2.10) we get

$$
\left[U(\cdot) T_{j} G_{\lambda}\right]\left(\mathbf{y}_{k}\right) \in H^{v}(\mathbb{R})
$$

for any $v<1 / 2$, as a function of $t$.

The last contribution to the inhomogeneous term in (1.13) is given by

$$
\begin{aligned}
{\left[U(t) T_{j} G_{\lambda}\right]\left(\mathbf{y}_{j}\right) } & =\frac{1}{2 \pi^{2}} \int_{0}^{\infty} d k \frac{k^{2}}{k^{2}+\lambda} \mathrm{e}^{-i k^{2} t} \\
& =\frac{\pi^{-3 / 2}}{4} \sqrt{\frac{-i}{t}}-\frac{\lambda}{4 \pi^{2}} \int_{0}^{\infty} d \omega \frac{\mathrm{e}^{-i \omega t}}{\sqrt{\omega}(\omega+\lambda)}
\end{aligned}
$$

which is finite for any $t \neq 0$.

First we show that the last term in (2.13) belongs to $L_{\text {loc }}^{2}(\mathbb{R})$. Indeed, for any $a>0$, applying Fubini-Tonelli theorem, we find

$$
\begin{aligned}
& \int_{-a}^{a} d t\left|\int_{0}^{\infty} d \omega \frac{\mathrm{e}^{-i \omega t}}{\sqrt{\omega}(\omega+\lambda)}\right|^{2} \\
& \quad=2 \int_{0}^{\infty} \frac{d \omega}{\sqrt{\omega}(\omega+\lambda)} \int_{0}^{\infty} \frac{d \omega^{\prime}}{\sqrt{\omega^{\prime}}\left(\omega^{\prime}+\lambda\right)} \frac{\sin \left[\left(\omega^{\prime}-\omega\right) a\right]}{\omega^{\prime}-\omega}<\infty .
\end{aligned}
$$

Moreover

$$
\int_{0}^{\infty} d \omega \frac{\omega^{2 \beta-1}}{(\omega+\lambda)^{2}}<\infty
$$


if $0<\beta<1$. This means that $\left[U(t) T_{j} G_{\lambda}\right]\left(\mathbf{y}_{j}\right)-\left(4 \pi^{3 / 2}\right)^{-1} \sqrt{-i / t}$ belongs to $H_{\mathrm{loc}}^{v}(\mathbb{R})$ for any $v<1$.

The inhomogeneous term in Eq. (1.13) can now be written as

$$
\begin{aligned}
\int_{0}^{t} d s \frac{\left(U(s) \psi_{0}\right)\left(\mathbf{y}_{j}\right)}{\sqrt{t-s}}= & \int_{0}^{t} d s \frac{\left(U(s) \phi_{0 \lambda}\right)\left(\mathbf{y}_{j}\right)}{\sqrt{t-s}}+\sum_{k \neq j} q_{k 0} \int_{0}^{t} d s \frac{\left[U(s) T_{k} G_{\lambda}\right]\left(\mathbf{y}_{j}\right)}{\sqrt{t-s}} \\
& -q_{j 0} \frac{\pi^{-3 / 2}}{4} \sqrt{-i} \int_{0}^{t} \frac{d s}{\sqrt{s(t-s)}} \\
& -q_{j 0} \frac{\lambda}{4 \pi^{2}} \int_{0}^{t} \frac{d s}{\sqrt{t-s}} \int_{0}^{\infty} d \omega \frac{\mathrm{e}^{-i \omega s}}{\sqrt{\omega}(\omega+\lambda)} .
\end{aligned}
$$

Using the explicit integral

$$
\int_{0}^{t} \frac{d s}{\sqrt{s(t-s)}}=\pi
$$

and Lemma 2 we conclude that (2.16) is an element of $H_{\text {loc }}^{v}(\mathbb{R})$, for any $v<1$. Moreover, due to the regularizing properties of the Abel kernel ([9]), it also belongs to $W_{\text {loc }}^{1,1}(\mathbb{R})$.

Step 2. The inhomogeneous term of the equation for $q_{j}(t)$ is a continuous function of $t$. Hence, using well-known results in the theory of nonlinear Volterra integral equations (see e.g. [12]), we deduce existence and uniqueness of the solution $q_{j}(t)$ of $(1.13)$ in the space $C^{0}([0, T])$, for $T>0$ sufficiently small.

Now, recalling that $\left.|| q_{j}\left(t_{1}\right)\right|^{2 \sigma_{j}} q_{j}\left(t_{1}\right)-\left|q_{j}\left(t_{2}\right)\right|^{2 \sigma_{j}} q_{j}\left(t_{2}\right)|\leqslant C| q_{j}\left(t_{1}\right)-q_{j}\left(t_{2}\right) \mid$ and exploiting Lemmas 2 and 3 we have that $q_{j} \in H^{v}(0, T)$ for any $v<1$. Moreover, from the smoothing properties of the Abel kernel in $W^{s, 1}$-spaces ([9]), we have that $q_{j} \in W^{1,1}(0, T)$.

Step 3. In Fourier space, Eq. (1.9) reads:

$$
\begin{aligned}
\hat{\psi}(t, \mathbf{k})= & \mathrm{e}^{-i k^{2} t} \hat{\psi}_{0}(\mathbf{k})+i \sum_{j=1}^{n} \mathrm{e}^{i \mathbf{k} \cdot \mathbf{y}_{j}} \int_{0}^{t} d s \mathrm{e}^{-i k^{2}(t-s)} q_{j}(s) \\
= & \mathrm{e}^{-i k^{2} t} \hat{\phi}_{0 \lambda}(\mathbf{k})+\mathrm{e}^{-i k^{2} t} \sum_{j=1}^{n} q_{j 0} \frac{\mathrm{e}^{i \mathbf{k} \cdot \mathbf{y}_{j}}}{k^{2}+\lambda} \\
& +i \mathrm{e}^{-i k^{2} t} \sum_{j=1}^{n} \mathrm{e}^{i \mathbf{k} \cdot \mathbf{y}_{j}} \int_{0}^{t} d s \mathrm{e}^{i\left(k^{2}+\lambda\right) s} \mathrm{e}^{-i \lambda s} q_{j}(s) \\
= & \mathrm{e}^{-i k^{2} t} \hat{\phi}_{0 \lambda}(\mathbf{k})+\sum_{j=1}^{n} q_{j}(t) \frac{\mathrm{e}^{i \mathbf{k} \cdot \mathbf{y}_{j}}}{k^{2}+\lambda}+\sum_{j=1}^{n} \mathrm{e}^{i \mathbf{k} \cdot \mathbf{y}_{j}}\left[\hat{f}_{1 \lambda}^{j}(t, \mathbf{k})+\hat{f}_{2 \lambda}^{j}(t, \mathbf{k})\right],
\end{aligned}
$$

where (2.20) is obtained integrating by parts in (2.19) and

$$
\hat{f}_{1 \lambda}^{j}(t, \mathbf{k})=\frac{i \lambda}{k^{2}+\lambda} \int_{0}^{t} d s \mathrm{e}^{-i k^{2}(t-s)} q_{j}(s),
$$




$$
\hat{f}_{2 \lambda}^{j}(t, \mathbf{k})=-\frac{1}{k^{2}+\lambda} \int_{0}^{t} d s \mathrm{e}^{-i k^{2}(t-s)} \dot{q}_{j}(s) .
$$

Now we compute the Sobolev degree of $f_{1 \lambda}^{j}(t)$ and $f_{2 \lambda}^{j}(t)$.

$$
\begin{aligned}
\int_{\mathbb{R}^{3}} d \mathbf{k}\left|k^{\beta} \hat{f}_{1 \lambda}^{j}(t, \mathbf{k})\right|^{2} & =4 \pi \lambda^{2} \int_{0}^{\infty} d k \frac{k^{2 \beta+2}}{\left(k^{2}+\lambda\right)^{2}}\left|\int_{0}^{t} d s \mathrm{e}^{i k^{2} s} q_{j}(s)\right|^{2} \\
& =2 \pi \lambda^{2} \int_{0}^{\infty} d \omega \frac{\omega^{\beta+1 / 2}}{(\omega+\lambda)^{2}}\left|\left(\mathcal{F} \tilde{q}_{j}^{0, t}\right)(\omega)\right|^{2} .
\end{aligned}
$$

Since $\tilde{q}_{j}^{0, t}$ has compact support its Fourier transform belongs to $C^{\infty}(\mathbb{R})$. From Lemma 3 we know that $\tilde{q}_{j}^{0, t} \in H^{v}(0, T)$ for any $v<1 / 2$ and therefore the integral in (2.24) converges for $-3 / 2<\beta<5 / 2$. It follows that $f_{1 \lambda}^{j}(t) \in H^{\mu}\left(\mathbb{R}^{3}\right)$ for any $\mu<5 / 2$.

Concerning $\hat{f}_{2 \lambda}^{j}$ we have:

$$
\begin{aligned}
\int_{\mathbb{R}^{3}} d \mathbf{k}\left|k^{\beta} \hat{f}_{2 \lambda}^{j}(t, \mathbf{k})\right|^{2} & =4 \pi \int_{0}^{\infty} d k \frac{k^{2 \beta+2}}{\left(k^{2}+\lambda\right)^{2}}\left|\int_{0}^{t} d s \mathrm{e}^{i k^{2} s} \dot{q}_{j}(s)\right|^{2} \\
& =2 \pi \int_{0}^{\infty} d \omega \frac{\omega^{\beta+1 / 2}}{(\omega+\lambda)^{2}}\left|\left(\mathcal{F}^{\tilde{q}}{ }^{0, t}\right)(\omega)\right|^{2} .
\end{aligned}
$$

Now, for any $t \in[0, T]$, let us consider the function $Q_{j}^{0, t}$ which extends $q_{j}:[0, t] \rightarrow \mathbb{C}$ in the following way: $Q_{j}^{0, t}(s)=q_{j 0}$ for any $s<0, Q_{j}^{0, t}(s)=q_{j}(t)$ for any $s>t$. Since $Q_{j}^{0, t}$ belongs to $H_{\text {loc }}^{v}(\mathbb{R})$ for any $v<1$ (see e.g. [10]), its derivative, which is compactly supported and coincides with $\tilde{\dot{q}}^{0, t}$, is an element of $H^{v}(\mathbb{R})$ for any $v<0$. Moreover, the Fourier transform of $\tilde{\dot{q}}_{j}^{0, t}$ belongs to $C^{\infty}(\mathbb{R})$. Therefore the integral in (2.26) converges if $-3 / 2<\beta<3 / 2$, so $f_{2 \lambda}^{j}(t) \in H^{\mu}\left(\mathbb{R}^{3}\right)$ for any $\mu<3 / 2$.

Hence, for any $t \in[0, T]$, we have $\phi_{\lambda}(t) \in H^{\mu}\left(\mathbb{R}^{3}\right)$ for $\mu<3 / 2$.

Remark 1. - The proof of Theorem 4 can be easily generalized to initial data with $\phi_{0 \lambda} \in H^{\rho}\left(\mathbb{R}^{3}\right), \rho>3 / 2$.

Remark 2. - We have the following alternative: either $\psi(t)$ is defined globally in time, or there exists $T<\infty$ such that $\limsup _{t \rightarrow T}\left|q_{j}(t)\right|=\infty$ for some $j$.

In any case there is a maximal time of existence $T^{*} \in(0,+\infty]$.

These are consequences of well-known results on continuation of solutions of nonlinear Volterra integral equations ([12]).

\section{The boundary condition}

In this section we investigate the relation between the evolution problem described by (1.8), (1.12), and its integral version (1.9) and (1.13) discussed in the previous section.

More precisely, we shall prove the following result: 
THEOREM 5. - The solution of the problem (1.9), (1.13), with an initial datum $\psi_{0}=$ $\phi_{0 \lambda}+\sum_{l=1}^{n} q_{l 0} G_{\lambda}\left(\cdot-\mathbf{y}_{l}\right), \phi_{0 \lambda} \in H^{2}\left(\mathbb{R}^{3}\right)$, satisfies

$$
\begin{aligned}
& \left(v, i \frac{\partial \psi}{\partial t}(t)\right)=(v,-\Delta \psi(t)) \quad v \in C_{0}^{\infty}\left(\mathbb{R}^{3} \backslash\left\{\mathbf{y}_{1}, \ldots, \mathbf{y}_{n}\right\}\right), \\
& \lim _{\mathbf{x} \rightarrow \mathbf{y}_{j}}\left(\psi(t, \mathbf{x})-\frac{q_{j}(t)}{4 \pi\left|\mathbf{x}-\mathbf{y}_{j}\right|}\right)=\gamma_{j}\left|q_{j}(t)\right|^{2 \sigma_{j}} q_{j}(t) \quad j=1, \ldots, n .
\end{aligned}
$$

Proof. - We know from Theorem 4 that $\psi(t)$ belongs to $V$ for any $t \in[0, T]$ and then in particular $\psi(t)$ is a distribution in $\mathbb{R}^{3}$.

In order to prove (3.1) it is sufficient to compute the distributional derivative of (2.18) with respect to time. We obtain

$$
i \frac{\partial}{\partial t} \hat{\psi}(t, \mathbf{k})=k^{2} \hat{\psi}(t, \mathbf{k})-\sum_{j=1}^{n} \mathrm{e}^{i \mathbf{k} \cdot \mathbf{y}_{j}} q_{j}(t)
$$

which in position space reads

$$
i \frac{\partial}{\partial t} \psi(t, \mathbf{x})=-\Delta \psi(t, \mathbf{x})-\sum_{j=1}^{n} q_{j}(t) \delta_{\mathbf{y}_{j}} .
$$

Then, for any $v \in C_{0}^{\infty}\left(\mathbb{R}^{3} \backslash\left\{\mathbf{y}_{1}, \ldots, \mathbf{y}_{n}\right\}\right)$, we obtain (3.1).

For the proof of (3.2), notice that

$$
\begin{aligned}
\psi(t, \mathbf{x})-\frac{q_{j}(t)}{4 \pi\left|\mathbf{x}-\mathbf{y}_{j}\right|}= & {\left[U(t) \psi_{0}\right](\mathbf{x})+i \int_{0}^{t} d s U\left(t-s ; \mathbf{x}-\mathbf{y}_{j}\right) q_{j}(s) } \\
& +i \sum_{l \neq j} \int_{0}^{t} d s U\left(t-s ; \mathbf{x}-\mathbf{y}_{l}\right) q_{l}(s)-\frac{q_{j}(t)}{4 \pi\left|\mathbf{x}-\mathbf{y}_{j}\right|}
\end{aligned}
$$

For the first term in the r.h.s. of (3.5) one has

$$
\lim _{\mathbf{x} \rightarrow \mathbf{y}_{j}}\left[U(t) \psi_{0}\right](\mathbf{x})=\lim _{\mathbf{x} \rightarrow \mathbf{y}_{j}}\left[U(t) \phi_{0 \lambda}\right](\mathbf{x})+\sum_{l=1}^{n} q_{0 l} \lim _{\mathbf{x} \rightarrow \mathbf{y}_{j}}\left[U(t) G_{\lambda}\left(\cdot-\mathbf{y}_{l}\right)\right](\mathbf{x}) .
$$

The first limit in the r.h.s. of (3.6) is trivial since $\nabla U(t) \phi_{0 \lambda}$ and $\Delta U(t) \phi_{0 \lambda}$ belong to $L^{2}\left(\mathbb{R}^{3}\right)$, therefore $U(t) \phi_{0 \lambda}$ is continuous at any $\mathbf{x}$.

Moreover we observe that the integral (see e.g. [7])

$$
\int_{0}^{\infty} d x \frac{\sin (a x)}{a x} \mathrm{e}^{i b x^{2}}=\frac{\sqrt{-\pi i}}{2 a} \int_{0}^{\frac{a}{\sqrt{b}}} d w \mathrm{e}^{i \frac{w^{2}}{4}}, \quad a \geqslant 0, b>0,
$$

is a continuous function of the parameter $a$. Then 


$$
\begin{aligned}
\lim _{\mathbf{x} \rightarrow \mathbf{y}_{j}}\left[U(t) G_{0}\left(\cdot-\mathbf{y}_{l}\right)\right](\mathbf{x}) & =\lim _{\mathbf{x} \rightarrow \mathbf{y}_{j}} \int_{0}^{\infty} \frac{d k}{2 \pi^{2}} \frac{\sin \left(k\left|\mathbf{x}-\mathbf{y}_{l}\right|\right)}{k\left|\mathbf{x}-\mathbf{y}_{l}\right|} \mathrm{e}^{-i k^{2} t} \\
& =\int_{0}^{\infty} \frac{d k}{2 \pi^{2}} \frac{\sin \left(k\left|\mathbf{y}_{j}-\mathbf{y}_{l}\right|\right)}{k\left|\mathbf{y}_{j}-\mathbf{y}_{l}\right|} \mathrm{e}^{-i k^{2} t} .
\end{aligned}
$$

Since the difference $\left[U(t) G_{\lambda}\left(\cdot-\mathbf{y}_{l}\right)\right](\mathbf{x})-\left[U(t) G_{0}\left(\cdot-\mathbf{y}_{l}\right)\right](\mathbf{x})$ is obviously continuous, we have

$$
\lim _{\mathbf{x} \rightarrow \mathbf{y}_{j}}\left[U(t) \psi_{0}\right](\mathbf{x})=\left[U(t) \psi_{0}\right]\left(\mathbf{y}_{j}\right) .
$$

Writing the second term in the r.h.s. of (3.5) in the Fourier space, one has

$$
\begin{aligned}
& i \int_{0}^{t} d s \mathrm{e}^{-i k^{2}(t-s)} q_{j}(s) \mathrm{e}^{i \mathbf{k} \cdot \mathbf{y}_{j}}-\frac{q_{j}(t)}{k^{2}} \mathrm{e}^{i \mathbf{k} \cdot \mathbf{y}_{j}} \\
& \quad=\mathrm{e}^{i \mathbf{k} \cdot \mathbf{y}_{j}}\left\{-\frac{\mathrm{e}^{-i k^{2} t}}{k^{2}} q_{j 0}-\int_{0}^{t} d s \frac{\mathrm{e}^{-i k^{2}(t-s)}}{k^{2}} \dot{q}(s)\right\} .
\end{aligned}
$$

Therefore,

$$
\begin{gathered}
\lim _{\mathbf{x} \rightarrow \mathbf{y}_{j}}\left\{i \int_{0}^{t} d s U\left(t-s ; \mathbf{x}-\mathbf{y}_{j}\right) q_{j}(s)-\frac{q_{j}(s)}{4 \pi\left|\mathbf{x}-\mathbf{y}_{j}\right|}\right\} \\
=\lim _{\mathbf{x} \rightarrow \mathbf{y}_{j}}(2 \pi)^{-3}\left\{-\int_{\mathbb{R}^{3}} d \mathbf{k} \frac{\mathrm{e}^{-i \mathbf{k} \cdot\left(\mathbf{x}-\mathbf{y}_{j}\right)}}{k^{2}} \mathrm{e}^{-i k^{2} t} q_{j 0}\right. \\
\left.-\int_{\mathbb{R}^{3}} d \mathbf{k} \mathrm{e}^{-i \mathbf{k} \cdot\left(\mathbf{x}-\mathbf{y}_{j}\right)} \int_{0}^{t} d s \frac{\mathrm{e}^{-i k^{2}(t-s)}}{k^{2}} \dot{q}_{j}(s)\right\} .
\end{gathered}
$$

For the first limit in the r.h.s. of (3.11) we have:

$$
\begin{aligned}
& -\frac{q_{j 0}}{4 \pi^{2}} \lim _{\mathbf{x} \rightarrow \mathbf{y}_{j}} \int_{0}^{\infty} d k \mathrm{e}^{-i k^{2} t} \int_{0}^{\pi} \mathrm{e}^{-i k\left|\mathbf{x}-\mathbf{y}_{j}\right| \cos \theta} \sin \theta d \theta \\
& =-\frac{q_{j 0}}{2 \pi^{2}} \lim _{\mathbf{x} \rightarrow \mathbf{y}_{j}} \int_{0}^{\infty} d k \mathrm{e}^{-i k^{2} t} \frac{2 \sin \left(k\left|\mathbf{x}-\mathbf{y}_{j}\right|\right)}{k\left|\mathbf{x}-\mathbf{y}_{j}\right|} \\
& =-\frac{q_{j 0}}{4 \pi^{3 / 2} \sqrt{i t}} .
\end{aligned}
$$

In the limit $\mathbf{x} \rightarrow \mathbf{y}_{j}$, the second integral in the r.h.s. of (3.11) equals

$$
-\frac{1}{2 \pi^{2}} \lim _{\mathbf{x} \rightarrow \mathbf{y}_{j}} \lim _{R \rightarrow \infty} \int_{0}^{t} d s \dot{q}_{j}(s) \int_{0}^{R} d k \mathrm{e}^{-i k^{2}(t-s)} \frac{\sin \left(k\left|\mathbf{x}-\mathbf{y}_{j}\right|\right)}{k\left|\mathbf{x}-\mathbf{y}_{j}\right|} .
$$


Since

$$
\lim _{R \rightarrow \infty} \int_{0}^{R} d k \mathrm{e}^{-i k^{2}(t-s)} \frac{\sin \left(k\left|\mathbf{x}-\mathbf{y}_{j}\right|\right)}{k\left|\mathbf{x}-\mathbf{y}_{j}\right|}=\frac{\sqrt{-\pi i}}{2\left|\mathbf{x}-\mathbf{y}_{j}\right|} \int_{0}^{\frac{\left|\mathbf{x}-\mathbf{y}_{j}\right|}{\sqrt{t-s}}} d w \mathrm{e}^{i w^{2} / 4}
$$

using Fubini-Tonelli theorem we interchange the limit $R \rightarrow \infty$ and the integration in the variable $s$. Furthermore one has

$$
\lim _{\mathbf{x} \rightarrow \mathbf{y}_{j}} \dot{q}_{j}(s) \int_{0}^{\infty} d k \frac{\sin \left(k\left|\mathbf{x}-\mathbf{y}_{j}\right|\right)}{k\left|\mathbf{x}-\mathbf{y}_{j}\right|} \mathrm{e}^{-i k^{2}(t-s)}=\frac{\sqrt{-i \pi}}{2 \sqrt{t-s}} \dot{q}_{j}(s) .
$$

Finally, applying the dominate convergence theorem, we obtain:

$$
-\lim _{\mathbf{x} \rightarrow \mathbf{y}_{j}} \int_{\mathbb{R}^{3}} d \mathbf{k} \mathrm{e}^{-i \mathbf{k} \cdot\left(\mathbf{x}-\mathbf{y}_{j}\right)} \int_{0}^{t} d s \frac{\mathrm{e}^{-i k^{2}(t-s)}}{k^{2}} \dot{q}_{j}(s)=-\frac{\sqrt{-i}}{4 \pi^{3 / 2}} \int_{0}^{t} d s \frac{\dot{q}_{j}(s)}{\sqrt{t-s}} .
$$

Now we use the Abel inversion formula:

$$
f(t)=\frac{1}{\pi} \frac{d}{d t} \int_{0}^{t} \frac{d \tau}{\sqrt{t-\tau}} \int_{0}^{\tau} d s \frac{f(s)}{\sqrt{\tau-s}}
$$

to obtain

$$
\begin{aligned}
\lim _{\mathbf{x} \rightarrow \mathbf{y}_{j}}\left\{\psi(t, \mathbf{x})-\frac{q_{j}(t)}{4 \pi\left|\mathbf{x}-\mathbf{y}_{j}\right|}\right\}= & \frac{1}{\pi} \frac{d}{d t} \int_{0}^{t} \frac{d \tau}{\sqrt{t-\tau}}\left\{\int_{0}^{\tau} d s \frac{\left[U(s) \psi_{0}\right]\left(\mathbf{y}_{j}\right)}{\sqrt{\tau-s}}\right. \\
& +i \sum_{l \neq j} \int_{0}^{\tau} d s \int_{0}^{s} d s^{\prime} \frac{U\left(s-s^{\prime} ; \mathbf{y}_{l}-\mathbf{y}_{j}\right)}{\sqrt{\tau-s}} q_{l}\left(s^{\prime}\right) \\
& -\frac{\sqrt{-i}}{4 \pi^{3 / 2}} q_{j 0} \int_{0}^{\tau} \frac{d s}{\sqrt{s(t-s)}} \\
& \left.-\frac{\sqrt{-i}}{4 \pi^{3 / 2}} \int_{0}^{\tau} \frac{d s}{\sqrt{\tau-s}} \int_{0}^{s} d s^{\prime} \frac{\dot{q}_{j}\left(s^{\prime}\right)}{\sqrt{s-s^{\prime}}}\right\} .
\end{aligned}
$$

We observe that a change of the integration variable yields

$$
\begin{aligned}
& \int_{0}^{\tau} d s \int_{0}^{s} d s^{\prime} \frac{\mathrm{e}^{i \frac{\left|\mathbf{y}_{l}-\mathbf{y}_{j}\right|^{2}}{4\left(s-s^{\prime}\right)}}}{\left[4 \pi i\left(s-s^{\prime}\right)\right]^{3 / 2}} \frac{q_{l}\left(s^{\prime}\right)}{\sqrt{\tau-s}} \\
& =\frac{1}{(4 \pi i)^{3 / 2}} \int_{0}^{\tau} d s^{\prime} \frac{q_{l}\left(s^{\prime}\right)}{\sqrt{\tau-s^{\prime}}} \mathrm{e}^{i \frac{\left|\mathbf{y}_{l}-\mathbf{y}_{j}\right|^{2}}{4\left(\tau-s^{\prime}\right)}} \int_{0}^{\infty} d v \frac{\mathrm{e}^{i \frac{\left|\mathbf{y}_{l}-\mathbf{y}_{j}\right|^{2} v}{4}}}{\sqrt{v}}
\end{aligned}
$$




$$
=\frac{1}{4 \pi i\left|\mathbf{y}_{l}-\mathbf{y}_{j}\right|} \int_{0}^{\tau} d s^{\prime} \frac{q_{l}\left(s^{\prime}\right)}{\sqrt{\tau-s^{\prime}}} \mathrm{e}^{i \frac{\left|\mathbf{y}_{l}-\mathbf{y}_{j}\right|^{2}}{4\left(\tau-s^{\prime}\right)}} .
$$

Moreover,

$$
\int_{0}^{\tau} \frac{d s}{\sqrt{\tau-s}} \int_{0}^{s} d s^{\prime} \frac{\dot{q}_{j}(s)}{\sqrt{s-s^{\prime}}}=\pi q_{j}(\tau)-\pi q_{j 0} .
$$

From (3.19), (3.20), (3.21) and (1.13) we conclude

$$
\begin{aligned}
\lim _{\mathbf{x} \rightarrow \mathbf{y}_{j}}\left\{\psi(t, \mathbf{x})-\frac{q_{j}(t)}{4 \pi\left|\mathbf{x}-\mathbf{y}_{j}\right|}\right\} & =\frac{1}{\pi} \frac{d}{d t} \int_{0}^{t} \frac{d \tau}{\sqrt{t-\tau}} \int_{0}^{\tau} d s \gamma_{j}\left|q_{j}(s)\right|^{2 \sigma_{j}} q_{j}(s) \\
& =\gamma_{j}\left|q_{j}(t)\right|^{2 \sigma_{j}} q_{j}(t) .
\end{aligned}
$$

\section{Local existence for initial data in $V$}

As a first step, we show that problem (1.9), (1.13) is well defined in $V$ and obtain a regularity result for the solution $\psi(t)$.

THEOREM 6. - Given $\psi_{0} \in V$, there exists $T>0$ such that problem (1.9), (1.13) has a unique solution $\psi(t) \in V, t \in[0, T]$.

Proof. - We follow step by step the analysis performed in Theorem 4.

Step 1. Using (2.5) and (2.6) the estimate (2.9) gives that $-1 / 4 \leqslant \beta \leqslant 1 / 4$, so $\left(U(\cdot) \phi_{0 \lambda}\right)\left(\mathbf{y}_{j}\right) \in H^{1 / 4}(\mathbb{R})$. Applying Abel transform, we obtain that the inhomogeneous term of Eq. (1.13) belongs to $H_{\text {loc }}^{3 / 4}(\mathbb{R})$.

Step 2. Since any function in $H_{\text {loc }}^{3 / 4}(\mathbb{R})$ is continuous, the proof of existence and uniqueness for the solution of (1.13) is identical to the proof given in Theorem 4.

Finally, the same bootstrap argument leads us to conclude that $q_{j} \in H_{\mathrm{loc}}^{3 / 4}(0, T)$.

Step 3. The fact that the regularity of the functions $q_{j}$ implies $\psi(t) \in V$ is proved in the same way as in Theorem 4.

From (2.20) it is clear that the Sobolev degree of $\phi_{\lambda}(t)$ cannot be larger than one if $\phi_{0 \lambda} \in H^{1}\left(\mathbb{R}^{3}\right)$.

Now we will show that the solution of the problem (1.9), (1.13) with initial datum in $V$ can be approximated by the solutions of the same problem with more regular initial data.

To this purpose, the first step consists in proving the following proposition.

Proposition 7. - Consider the function $\psi_{0}=\phi_{0 \lambda}+\sum_{j=1}^{n} q_{j 0} T_{j} G_{\lambda}$ and the sequence $\psi_{0}^{(l)}=\phi_{0 \lambda}^{(l)}+\sum_{j=1}^{n} q_{j 0}^{(l)} T_{j} G_{\lambda}$ with $\phi_{0 \lambda}^{(l)} \in H^{2}\left(\mathbb{R}^{3}\right), \phi_{0 \lambda}^{(l)} \rightarrow \phi_{0 \lambda}$ in $H^{1}\left(\mathbb{R}^{3}\right)$ for $l \rightarrow \infty, q_{j 0}^{(l)} \rightarrow q_{j 0}$ for $l \rightarrow \infty$.

Moreover let $q_{j}, q_{j}^{(l)}$ be the solution of (1.13) corresponding to the initial data $\psi_{0}$, $\psi_{0}^{(l)}$ respectively.

Then, for $l \rightarrow \infty, q_{j}^{(l)}$ converges to $q_{j}$ in $H^{3 / 4}(0, T), T>0$. 
Proof. - Following the line of Lemma 12 in [2], we first observe that, due to the properties of Abel integrals, and to the boundedness of $q_{j}$,

$$
\left\|\int_{0} d s \frac{\left|q_{j}^{(l)}(s)\right|^{2 \sigma_{j}} q_{j}^{(l)}(s)-\left|q_{j}(s)\right|^{2 \sigma_{j}} q_{j}(s)}{\sqrt{\cdot-s}}\right\|_{H^{3 / 4}(0, T)} \leqslant C(T)\left\|q_{j}^{(l)}-q_{j}\right\|_{H^{3 / 4}(0, T)}
$$

where $C(T)$ vanishes when $T$ goes to zero.

Analogously

$$
\left\|\int_{0} d s \frac{q_{k}^{(l)}(s)-q_{k}(s)}{\sqrt{\cdot-s}} \mathrm{e}^{i \frac{\left|\mathbf{y}_{j}-\mathbf{y}_{l}\right|^{2}}{-s}}\right\|_{H^{3 / 4}(0, T)} \leqslant C(T)\left\|q_{k}^{(l)}-q_{k}\right\|_{H^{3 / 4}(0, T)} .
$$

Now we analyze the inhomogeneous term in (1.13):

$$
\begin{aligned}
\int_{0}^{t} d s \frac{\left[U(s)\left(\psi_{0}^{(l)}-\psi_{0}\right)\right]\left(\mathbf{y}_{j}\right)}{\sqrt{t-s}}= & \int_{0}^{t} d s \frac{\left[U(s)\left(\phi_{0 \lambda}^{(l)}-\phi_{0 \lambda}\right)\right]\left(\mathbf{y}_{j}\right)}{\sqrt{t-s}} \\
& +\sum_{j=1}^{n}\left(q_{0 j}^{(l)}-q_{0 j}\right) \int_{0}^{t} d s \frac{\left[U(s) T_{j} G_{\lambda}\right]\left(\mathbf{y}_{j}\right)}{\sqrt{t-s}} .
\end{aligned}
$$

The last term in (4.3) is clearly vanishing in $H^{3 / 4}(0, T)$ as $l$ goes to infinity, while for the first term we obtain the same result by exploiting (2.9).

Now, choosing $T$ such that $C(T)$ in (4.1) is less than $(2 n)^{-1}$ one has

$$
\begin{aligned}
\max _{j}\left\|q_{j}^{(l)}-q_{j}\right\|_{H^{3 / 4}(0, T)} \leqslant & C \max _{j}\left\|\int_{0} d s \frac{\left[U(s)\left(\phi_{0 \lambda}^{(l)}-\phi_{0 \lambda}\right)\right]\left(\mathbf{y}_{j}\right)}{\sqrt{\cdot-s}}\right\|_{H^{3 / 4}(0, T)} \\
& +C \sum_{k=1}^{n}\left|q_{0 k}^{(l)}-q_{0 k}\right|
\end{aligned}
$$

completing the proof.

As a last step, we prove that the convergence of the $q^{(l)}$ 's implies the convergence of the $\psi^{(l)}$ 's.

THEOREM 8.-Given the function $\psi_{0}=\phi_{0 \lambda}+\sum_{j=1}^{n} q_{j 0} T_{j} G_{\lambda}$ and the sequence $\psi_{0}=\phi_{0 \lambda}^{(l)}+\sum_{j=1}^{n} q_{j 0}^{(l)} T_{j} G_{\lambda}$ with $\phi_{0 \lambda}^{(l)} \in H^{2}\left(\mathbb{R}^{3}\right), \phi_{0 \lambda}^{(l)} \rightarrow \phi_{0 \lambda}$ in $H^{1}\left(\mathbb{R}^{3}\right)$ for $l \rightarrow \infty$, $q_{j 0}^{(l)} \rightarrow q_{j 0}$ for $l \rightarrow \infty$, then the sequence of $\psi^{(l)}(t)$, solution of problem (1.9), (1.13), with initial datum $\psi_{0}^{(l)}$, converges in $V$ to $\psi(t)$, which solves the same problem with initial datum $\psi_{0}$, for any $t \in[0, T), T>0$.

Proof. - Following step 3 in the proof of Theorem 4, one has

$$
\begin{aligned}
\hat{\psi}^{(l)}(t, \mathbf{k})-\hat{\psi}(t, \mathbf{k})= & \mathrm{e}^{-i k^{2} t}\left[\hat{\phi}_{0 \lambda}^{(l)}(t, \mathbf{k})-\hat{\phi}_{0 \lambda}(t, \mathbf{k})\right] \\
& +\sum_{j=1}^{n}\left[q_{j}^{(l)}(t)-q_{j}(t)\right] \frac{\mathrm{e}^{i \mathbf{k} \cdot \mathbf{y}_{j}}}{k^{2}+\lambda}
\end{aligned}
$$




$$
+\sum_{j=1}^{n} \mathrm{e}^{i \mathbf{k} \cdot \mathbf{y}_{j}}\left[\hat{f}_{1 \lambda}^{j(l)}(t, \mathbf{k})+\hat{f}_{2 \lambda}^{j(l)}(t, \mathbf{k})\right]
$$

where

$$
\begin{aligned}
& \hat{f}_{1 \lambda}^{j(l)}(t, \mathbf{k})=\frac{i \lambda}{k^{2}+\lambda} \int_{0}^{t} d s \mathrm{e}^{-i k^{2}(t-s)}\left[q_{j}^{(l)}(s)-q_{j}(s)\right], \\
& \hat{f}_{2 \lambda}^{j(l)}(t, \mathbf{k})=-\frac{1}{k^{2}+\lambda} \int_{0}^{t} d s \mathrm{e}^{-i k^{2}(t-s)}\left[\dot{q}_{j}^{(l)}(s)-\dot{q}_{j}(s)\right] .
\end{aligned}
$$

Given the convergence of the $q_{j}^{(l)}(t)$, to prove the convergence of $\psi^{(l)}$ in $V$ we have to prove the convergence in $H^{1}\left(\mathbb{R}^{3}\right)$ of $\phi_{\lambda}^{(l)}(t)$. For this purpose it is sufficient to note that from (2.24) and (2.26) one has

$$
\begin{aligned}
& \int_{\mathbb{R}^{3}} d \mathbf{k}\left|k^{\beta} f_{1 \lambda}^{j(l)}(t, \mathbf{k})\right|^{2}=2 \pi \lambda^{2} \int_{0}^{\infty} d \omega \frac{\omega^{\beta+1 / 2}}{(\omega+\lambda)^{2}}\left|\left[\mathcal{F}\left(\tilde{q}_{j}^{(l) 0, t}-\tilde{q}_{j}^{0, t}\right)\right](\omega)\right|^{2}, \\
& \int_{\mathbb{R}^{3}} d \mathbf{k}\left|k^{\beta} f_{2 \lambda}^{j(l)}(t, \mathbf{k})\right|^{2}=2 \pi \int_{0}^{\infty} d \omega \frac{\omega^{\beta+1 / 2}}{(\omega+\lambda)^{2}}\left|\left[\mathcal{F}\left(\tilde{\dot{q}}_{j}^{(l) 0, t}-\tilde{\dot{q}}_{j}{ }^{0, t}\right)\right](\omega)\right|^{2} .
\end{aligned}
$$

From Proposition 7 and step 3 of Theorem 4 we obtain that for $\beta=1$ in the limit $l \rightarrow \infty$ the integrals in (4.8) and (4.9) vanish.

\section{Conservation laws}

First we prove the conservation law of the $L^{2}$-norm.

THEOREM 9. - Consider $\psi_{0}=\phi_{0 \lambda}+\sum_{k=1}^{n} q_{k 0} G_{\lambda}\left(\cdot-\mathbf{y}_{k}\right), \phi_{0 \lambda} \in H^{1}\left(\mathbb{R}^{3}\right)$, and let $\psi(t)$ be the corresponding solution of problem (1.9) in the maximal time interval $\left[0, T^{*}\right)$. Then $\|\psi(t)\|_{L^{2}\left(\mathbb{R}^{3}\right)}=\left\|\psi_{0}\right\|_{L^{2}\left(\mathbb{R}^{3}\right)}$ for any $t \in\left[0, T^{*}\right)$.

Proof. - From (2.19)

$$
\begin{aligned}
\|\hat{\psi}(t)\|_{L^{2}\left(\mathbb{R}^{3}\right)}^{2}= & \left\|\hat{\psi}_{0}\right\|_{L^{2}\left(\mathbb{R}^{3}\right)}^{2}+\sum_{j=1}^{n} \sum_{l \neq j} \int_{\mathbb{R}^{3}} d \mathbf{k} \mathrm{e}^{i \mathbf{k} \cdot\left(\mathbf{y}_{j}-\mathbf{y}_{l}\right)} \int_{0}^{t} d s \int_{0}^{t} d s^{\prime} \mathrm{e}^{i k^{2}\left(s-s^{\prime}\right)} q_{j}(s) \overline{q_{l}\left(s^{\prime}\right)} \\
& -2 \mathfrak{s} \sum_{j=1}^{n} \int_{\mathbb{R}^{3}} d \mathbf{k} \mathrm{e}^{i k^{2} t} \overline{\hat{\psi}_{0}(\mathbf{k})} \mathrm{e}^{i \mathbf{k} \cdot \mathbf{y}_{j}} \int_{0}^{t} d s \mathrm{e}^{-i k^{2}(t-s)} q_{j}(s) \\
= & (\mathrm{I})+(\mathrm{II})+(\mathrm{III}) .
\end{aligned}
$$

We want to show that (II) + (III) $=0$. In fact,

$$
\text { (II) }=2 \Re \sum_{j=1}^{n} \int_{\mathbb{R}^{3}} d \mathbf{k} \int_{0}^{t} d s \int_{0}^{s} d s^{\prime} \mathrm{e}^{i k^{2}\left(s-s^{\prime}\right)} q_{j}(s) \overline{q_{j}\left(s^{\prime}\right)}
$$




$$
\begin{aligned}
& +2 \Re \sum_{j=1}^{n} \sum_{l \neq j} \int_{\mathbb{R}^{3}} d \mathbf{k} \mathrm{e}^{i \mathbf{k} \cdot\left(\mathbf{y}_{j}-\mathbf{y}_{l}\right)} \int_{0}^{t} d s \int_{0}^{s} d s^{\prime} \mathrm{e}^{i k^{2}\left(s-s^{\prime}\right)} q_{j}(s) \overline{q_{l}\left(s^{\prime}\right)} \\
= & \left(\mathrm{II}_{d}\right)+\left(\mathrm{II}_{n d}\right) .
\end{aligned}
$$

Let us compute separately the diagonal and the non diagonal terms in (5.2)

$$
\begin{aligned}
\left(\mathrm{II}_{d}\right)= & -2 \Im \sum_{j=1}^{n} \int_{\mathbb{R}^{3}} d \mathbf{k} \frac{1}{k^{2}} \int_{0}^{t} d s \int_{0}^{s} d s^{\prime} \overline{q_{j}\left(s^{\prime}\right)} q_{j}(s) \frac{d}{d s^{\prime}} \mathrm{e}^{i k^{2}\left(s-s^{\prime}\right)} \\
= & 4 \pi^{3 / 2} \Im \sqrt{i} \sum_{j=1}^{n} \overline{q_{j 0}} \int_{0}^{t} d s \frac{q_{j}(s)}{\sqrt{s}} \\
& +4 \pi^{3 / 2} \Im \sqrt{i} \sum_{j=1}^{n} \int_{0}^{t} d s \int_{0}^{s} d s^{\prime} \frac{q_{j}(s) \overline{\dot{q}_{j}\left(s^{\prime}\right)}}{\sqrt{s-s^{\prime}}},
\end{aligned}
$$

where we have integrated by parts in $s^{\prime}$ and exchanged the integrals in $s$ and $s^{\prime}$ with the integral in $\mathbf{k}$ exploiting the dominated convergence theorem.

Concerning the non diagonal term we have

$$
\begin{aligned}
\left(\mathrm{II}_{n d}\right)= & 2 \Im \sum_{j=1}^{n} \sum_{l \neq j} \int_{0}^{t} d s \int_{0}^{s} d s^{\prime} q_{j}(s) \overline{q_{l}\left(s^{\prime}\right)} \frac{d}{d s} \int_{\mathbb{R}^{3}} \frac{d \mathbf{k}}{k^{2}} \mathrm{e}^{i k^{2}\left(s-s^{\prime}\right)} \mathrm{e}^{i \mathbf{k} \cdot\left(\mathbf{y}_{j}-\mathbf{y}_{l}\right)} \\
= & 8 \pi \Im \sum_{j=1}^{n} \sum_{l \neq j} \frac{1}{\left|\mathbf{y}_{j}-\mathbf{y}_{l}\right|} \int_{0}^{s} d s \int_{0}^{s} d s^{\prime} q_{j}(s) \overline{q_{l}\left(s^{\prime}\right)} \\
& \times \frac{d}{d s} \int_{0}^{\infty} \frac{d k}{k} \mathrm{e}^{i k^{2\left(s-s^{\prime}\right)}} \sin \left(k\left|\mathbf{y}_{j}-\mathbf{y}_{l}\right|\right) .
\end{aligned}
$$

From (3.15),

$$
\begin{aligned}
\left(\mathrm{II}_{n d}\right)= & 2 \pi^{3 / 2} \mathfrak{s} \sqrt{i} \sum_{j=1}^{n} \sum_{l \neq j} \int_{0}^{t} d s \int_{0}^{s} d s^{\prime} q_{j}(s) \overline{q_{l}\left(s^{\prime}\right)} \\
& \times \frac{d}{d s} \int_{s}^{+\infty} d \tau \frac{\exp \left(-i \frac{\left|\mathbf{y}_{j}-\mathbf{y}_{l}\right|^{2}}{4\left(\tau-s^{\prime}\right)}\right)}{\left(\tau-s^{\prime}\right)^{3 / 2}} \\
= & -2 \pi^{3 / 2} \Im \sqrt{i} \sum_{j=1}^{n} \sum_{l \neq j} \int_{0}^{t} d s \int_{0}^{s} d s^{\prime} q_{j}(s) \overline{q_{l}\left(s^{\prime}\right)} \frac{\exp \left(-i \frac{\mathbf{y}_{j}-\left.\mathbf{y}_{l}\right|^{2}}{4\left(s-s^{\prime}\right)}\right)}{\left(s-s^{\prime}\right)^{3 / 2}}
\end{aligned}
$$

Let us analyse (III). From (1.13) we have

$$
(\mathrm{III})=-16 \pi^{3} \Im \sum_{j=1}^{n} \int_{0}^{t} d s q_{j}(s) \overline{\left[U(s) \psi_{0}\right]\left(\mathbf{y}_{j}\right)}
$$




$$
\begin{aligned}
= & -4 \pi^{3 / 2} \Im \sum_{j=1}^{n} \sqrt{i} \int_{0}^{t} d s q_{j}(s) \frac{d}{d s} \int_{0}^{s} d s^{\prime} \frac{\overline{q_{j}\left(s^{\prime}\right)}}{\sqrt{s-s^{\prime}}} \\
& +4 \pi \Im \sum_{j=1}^{n} \sum_{j \neq l} \frac{1}{\left|\mathbf{y}_{j}-\mathbf{y}_{l}\right|} \int_{0}^{t} d s q_{j}(s) \frac{d}{d s} \int_{0}^{s} \frac{d s^{\prime}}{\sqrt{s-s^{\prime}}} \int_{0}^{s^{\prime}} d \tau \frac{\exp \left(-i \frac{\mid \mathbf{y}_{j}-\mathbf{y} l^{2}}{4\left(s^{\prime}-\tau\right)}\right)}{\sqrt{s^{\prime}-\tau}} \overline{q_{l}(\tau)} \\
& -16 \pi^{3} \Im \sum_{j=1}^{n} \gamma_{j} \int_{0}^{t} d s\left|q_{j}(s)\right|^{2 \sigma_{j}+2} \\
= & \left(\mathrm{III}_{d}\right)+\left(\mathrm{III}_{n d}\right)+(\mathrm{IV}) .
\end{aligned}
$$

Note that $(\mathrm{IV})=0$. Moreover

$$
\begin{aligned}
\left(\mathrm{III}_{d}\right)= & -4 \pi^{3 / 2} \mathfrak{s} \sqrt{i} \sum_{j=1}^{n} \overline{q_{j 0}} \int_{0}^{t} d s \frac{q_{j}(s)}{\sqrt{s}} \\
& -4 \pi^{3 / 2} \mathfrak{s} \sqrt{i} \sum_{j=1}^{n} \int_{0}^{t} d s \int_{0}^{s} d s^{\prime} \frac{q_{j}(s) \overline{\dot{q}_{j}\left(s^{\prime}\right)}}{\sqrt{s-s^{\prime}}} .
\end{aligned}
$$

Then, from (5.4), $\left(\mathrm{II}_{d}\right)+\left(\mathrm{III}_{d}\right)=0$.

Concerning $\left(\mathrm{III}_{n d}\right)$, it is sufficient to recall the Abel inversion formula (3.18) and formula (3.20) to obtain

$$
\left(\mathrm{III}_{n d}\right)=2 \pi^{3 / 2} \Im \sqrt{i} \sum_{j=1}^{n} \sum_{l \neq j} \int_{0}^{t} d s q_{j}(s) \int_{0}^{s} d s^{\prime} \frac{\exp \left(-i \frac{\left|\mathbf{y}_{j}-\mathbf{y}_{l}\right|^{2}}{4\left(s-s^{\prime}\right)}\right)}{\left(s-s^{\prime}\right)^{3 / 2}} \overline{q_{l}\left(s^{\prime}\right)} .
$$

Since $\left(\mathrm{II}_{n d}\right)+\left(\mathrm{III}_{n d}\right)=0$, the proof is complete.

The next step is to define the energy of a solution of our nonlinear evolution problem and then to prove the corresponding conservation law.

DEFINITION 10. - For any $\psi \in V$, the energy of $\psi$ is defined by:

$$
E(\psi)=\|\nabla \phi\|_{L^{2}\left(\mathbb{R}^{3}\right)}^{2}+\sum_{k=1}^{n} \frac{\gamma_{k}}{\sigma_{k}+1}\left|q_{k}\right|^{2 \sigma_{k}+2}-\sum_{k<l=1}^{n} \frac{\Re\left(q_{k} \overline{q_{l}}\right)}{2 \pi\left|\mathbf{y}_{k}-\mathbf{y}_{l}\right|} .
$$

THEOREM 11. - Consider $\psi_{0} \in V$ and let $\psi(t)$ be the corresponding solution at time $t$. Then we have $E\left(\psi_{0}\right)=E(\psi(t))$.

Proof. - We compute $\|\nabla \phi(t)\|_{L^{2}\left(\mathbb{R}^{3}\right)}^{2}$ in the Fourier space. An integration by parts yields

$$
\begin{aligned}
\hat{\phi}(t, \mathbf{k}) & =\hat{\psi}(t, \mathbf{k})-\sum_{j=1}^{n} \frac{q_{j}(t)}{k^{2}} \mathrm{e}^{i \mathbf{k} \cdot \mathbf{y}_{j}} \\
& =\mathrm{e}^{-i k^{2} t} \hat{\phi}_{0}(\mathbf{k})-\sum_{j=1}^{n} \int_{0}^{t} d s \frac{\exp \left[-i k^{2}(t-s)\right]}{k^{2}} \mathrm{e}^{i \mathbf{k} \cdot \mathbf{y}_{j}} \dot{q}_{j}(s) .
\end{aligned}
$$


Then

$$
\begin{aligned}
\|\widehat{\nabla \phi(t)}\|_{L^{2}\left(\mathbb{R}^{3}\right)}^{2}= & \left\|\widehat{\nabla \phi_{0}}\right\|_{L^{2}\left(\mathbb{R}^{3}\right)}^{2} \\
& +\sum_{j=1}^{n} \sum_{l \neq j} \int_{\mathbb{R}^{3}} \frac{d \mathbf{k}}{k^{2}} \mathrm{e}^{i \mathbf{k} \cdot\left(\mathbf{y}_{j}-\mathbf{y}_{l}\right)} \int_{0}^{t} d s \int_{0}^{t} d s^{\prime} \mathrm{e}^{i k^{2}\left(s-s^{\prime}\right)} \dot{q}_{j}(s) \overline{\dot{q}_{l}\left(s^{\prime}\right)} \\
& -16 \pi^{3} \Re \sum_{j=1}^{n} \int_{0}^{t} d s \dot{q}_{j}(s) \overline{\left[U(s) \phi_{0}\right]\left(\mathbf{y}_{j}\right)} \\
= & (\mathrm{V})+(\mathrm{VI})+(\mathrm{VII}) .
\end{aligned}
$$

As in the proof of Theorem 9 we study (VI) and (VII) separating the diagonal and nondiagonal terms. Let us define

$$
(\mathrm{VI})=\left(\mathrm{VI}_{d}\right)+\left(\mathrm{VI}_{n d}\right),
$$

where

$$
\left(\mathrm{VI}_{d}\right)=4 \pi^{3 / 2} \Re \sqrt{i} \sum_{j=1}^{n} \int_{0}^{t} d s \int_{0}^{s} d s^{\prime} \frac{\dot{q}_{j}(s) \overline{\dot{q}_{j}\left(s^{\prime}\right)}}{\sqrt{s-s^{\prime}}} .
$$

Moreover, exchanging the integral in $\mathbf{k}$ and in $s$ by using Lebesgue theorem, and applying formula (3.15), we find

$$
\begin{aligned}
\left(\mathrm{VI}_{n d}\right)= & 4 \pi^{3 / 2} \Re \sqrt{i} \sum_{j=1}^{n} \sum_{l \neq j} \frac{1}{\left|\mathbf{y}_{j}-\mathbf{y}_{l}\right|} \int_{0}^{t} d s \int_{0}^{s} d s^{\prime} \dot{q}_{j}(s) \overline{\dot{q}_{l}\left(s^{\prime}\right)} \int_{0}^{\frac{\left|\mathbf{y}_{j}-\mathbf{y}_{l}\right|}{\sqrt{s-s^{\prime}}}} d w \mathrm{e}^{-i \frac{w^{2}}{4}} \\
= & 2 \pi^{3 / 2} \Re \sqrt{i} \sum_{j=1}^{n} \sum_{l \neq j} \int_{0}^{t} d s \int_{0}^{s} d s^{\prime} \dot{q}_{j}(s) \overline{\dot{q}_{l}\left(s^{\prime}\right)} \int_{-\infty}^{s^{\prime}} d \tau \frac{\exp \left(-i \frac{\left|\mathbf{y}_{j}-\mathbf{y}_{l}\right|^{2}}{4(s-\tau)}\right)}{(s-\tau)^{3 / 2}} \\
= & 2 \pi^{3 / 2} \Re \sqrt{i} \sum_{j=1}^{n} \sum_{l \neq j} \int_{0}^{t} d s \dot{q}_{j}(s) \overline{\dot{q}_{l}(s)} \int_{-\infty}^{s} d \tau \frac{\exp \left(-i \frac{\left|\mathbf{y}_{j}-\mathbf{y}_{l}\right|^{2}}{4(s-\tau)}\right)}{(s-\tau)^{3 / 2}} \\
& -2 \pi^{3 / 2} \Re \sqrt{i} \sum_{j=1}^{n} \sum_{l \neq j} \overline{q_{l 0}} \int_{0}^{t} d s \dot{q}_{j}(s) \int_{-\infty}^{0} d \tau \frac{\exp \left(-i \frac{\left|\mathbf{y}_{j}-\mathbf{y}_{l}\right|^{2}}{4(s-\tau)}\right)}{(s-\tau)^{3 / 2}} \\
& -2 \pi^{3 / 2} \Re \sqrt{i} \sum_{j=1}^{n} \sum_{l \neq j} \int_{0}^{s} d s \int_{0}^{s} d s^{\prime} \dot{q}_{j}(s) \frac{\exp \left(-i \frac{\left|\mathbf{y}_{j}-\mathbf{y}_{l}\right|^{2}}{4\left(s-s^{\prime}\right)}\right)}{\left(s-s^{\prime}\right)^{3 / 2}} .
\end{aligned}
$$

Let us consider the first term in the r.h.s. of (5.17), which we denote (VI)'.

An explicit computation gives

$$
\int_{-\infty}^{s} d \tau \frac{\exp \left(-i \frac{\left|\mathbf{y}_{j}-\mathbf{y}_{l}\right|^{2}}{4(s-\tau)}\right)}{(s-\tau)^{3 / 2}}=2 \int_{0}^{+\infty} d z \mathrm{e}^{-i \frac{\left|\mathbf{y}_{j}-\mathbf{y}_{l}\right|^{2}}{4}} z^{2}=\frac{2 \sqrt{-\pi i}}{\left|\mathbf{y}_{j}-\mathbf{y}_{l}\right|}
$$


Therefore

$$
\begin{aligned}
(\mathrm{VI})^{\prime}= & 4 \pi^{2} \Re \sum_{j=1}^{n} \sum_{l \neq j} \frac{1}{\left|\mathbf{y}_{j}-\mathbf{y}_{l}\right|} \int_{0}^{t} d s \dot{q}_{j}(s) \overline{q_{l}(s)} \\
= & 4 \pi^{2} \Re \sum_{j=1}^{n} \sum_{l<j} \frac{1}{\left|\mathbf{y}_{j}-\mathbf{y}_{l}\right|} \int_{0}^{t} d s \dot{q}_{j}(s) \overline{q_{l}(s)} \\
& +4 \pi^{2} \Re \sum_{j=1}^{n} \sum_{l>j} \frac{1}{\left|\mathbf{y}_{j}-\mathbf{y}_{l}\right|} \int_{0}^{t} d s \dot{q}_{j}(s) \overline{q_{l}(s)} .
\end{aligned}
$$

Exchanging the role of the indices $l$ and $j$ and taking the complex conjugate of the second term in the r.h.s. of (5.19) one has

$$
\begin{aligned}
(\mathrm{VI})^{\prime} & =4 \pi^{2} \Re \sum_{j=1}^{n} \sum_{l \neq j} \frac{1}{\left|\mathbf{y}_{j}-\mathbf{y}_{l}\right|} \int_{0}^{t} d s\left[\dot{q}_{j}(s) \overline{q_{l}(s)}+\overline{\dot{q}_{l}(s)} q_{j}(s)\right] \\
& =4 \pi^{2} \Re \sum_{j=1}^{n} \sum_{l \neq j} \frac{1}{\left|\mathbf{y}_{j}-\mathbf{y}_{l}\right|} \int_{0}^{t} d s\left[q_{j}(t) \overline{q_{l}(t)}-q_{j 0} \overline{q_{l 0}}\right] .
\end{aligned}
$$

Now let us analyze (VII) in Eq. (5.14).

Since

$$
\begin{aligned}
{\left[U(s) \phi_{0}\right]\left(\mathbf{y}_{j}\right)=} & {\left[U(s) \psi_{0}\right]\left(\mathbf{y}_{j}\right)-\frac{\sqrt{-i}}{4 \pi^{3 / 2}} \frac{q_{j 0}}{\sqrt{s}} } \\
& -\frac{\sqrt{-i}}{4 \pi^{3 / 2}} \sum_{l \neq j} \frac{q_{l 0}}{\left|\mathbf{y}_{j}-\mathbf{y}_{l}\right|} \int_{0}^{\frac{\left|\mathbf{y}_{j}-\mathbf{y}_{l}\right|}{\sqrt{s}}} d w \mathrm{e}^{i w^{2} / 4}
\end{aligned}
$$

from (1.13) we find

$$
\begin{aligned}
& (\mathrm{VII})=-4 \pi^{3 / 2} \Re \sqrt{i} \sum_{j=1}^{n} \int_{0}^{t} d s \dot{q}_{j}(s) \frac{d}{d s} \int_{0}^{s} d s^{\prime} \frac{\overline{q_{j}\left(s^{\prime}\right)}}{\sqrt{s-s^{\prime}}} \\
& +4 \pi \Re \sum_{j=1}^{n} \sum_{l \neq j} \frac{1}{\left|\mathbf{y}_{j}-\mathbf{y}_{l}\right|} \int_{0}^{t} d s \dot{q}_{j}(s) \frac{d}{d s} \int_{0}^{s} \frac{d s^{\prime}}{\sqrt{s-s^{\prime}}} \int_{0}^{s^{\prime}} d \tau \frac{\exp \left(-i \frac{\left|\mathbf{y}_{j}-\mathbf{y}_{l}\right|^{2}}{4\left(s^{\prime}-\tau\right)}\right)}{\sqrt{s^{\prime}-\tau}} \overline{q_{l}(\tau)} \\
& +4 \pi^{3 / 2} \Re \sqrt{i} \sum_{j=1}^{n} \overline{q_{j 0}} \int_{0}^{t} d s \frac{\dot{q}_{j}(s)}{\sqrt{s}} \\
& +4 \pi^{3 / 2} \Re \sqrt{i} \sum_{j=1}^{n} \sum_{l \neq j} \int_{0}^{t} d s \dot{q}_{j}(s) \int_{0}^{\frac{\left|\mathbf{y}_{j}-\mathbf{y}_{l}\right|}{\sqrt{s}}} d w \mathrm{e}^{-i w^{2} / 4}
\end{aligned}
$$




$$
\begin{aligned}
& -16 \pi^{3} \Re \sum_{j=1}^{n} \int_{0}^{t} d s \dot{q}_{j}(s)\left|q_{j}(s)\right|^{2 \sigma_{j}} \overline{q_{j}(s)} \\
= & \left(\mathrm{VII}_{d}\right)+\left(\mathrm{VII}_{n d}\right)+(\mathrm{VIII})+(\mathrm{IX})+(\mathrm{X}) .
\end{aligned}
$$

Let us consider $\left(\mathrm{VII}_{d}\right)$. Performing the derivative in the variable $s$ one obtains

$$
\left(\mathrm{VII}_{d}\right)+\left(\mathrm{VI}_{d}\right)+(\mathrm{VIII})=0 .
$$

Concerning the nondiagonal terms, from formula (3.18) it is clear that

$$
\left(\mathrm{VII}_{n d}\right)=2 \pi^{3 / 2} \Re \sqrt{i} \sum_{j=1}^{n} \sum_{l \neq j} \int_{0}^{t} d s \int_{0}^{s} d s^{\prime} \frac{\exp \left(-i \frac{\left|\mathbf{y}_{j}-\mathbf{y}_{l}\right|^{2}}{4\left(s-s^{\prime}\right)}\right)}{\left(s-s^{\prime}\right)^{3 / 2}} \dot{q}_{j}(s) \overline{q_{l}\left(s^{\prime}\right)} .
$$

Moreover, after a change of variable, one has

$$
(\mathrm{IX})=2 \pi^{3 / 2} \Re \sqrt{i} \sum_{j=1}^{n} \sum_{l \neq j} \overline{q_{l 0}} \int_{0}^{t} d s \dot{q}_{j}(s) \int_{-\infty}^{0} d \tau \frac{\exp \left(-i \frac{\left|\mathbf{y}_{j}-\mathbf{y}_{l}\right|^{2}}{4(s-\tau)}\right)}{(s-\tau)^{3 / 2}} .
$$

From Eqs. (5.25), (5.26), (5.17), (5.20), we conclude

$$
\left(\mathrm{VI}_{n d}\right)+\left(\mathrm{VII}_{n d}\right)+(\mathrm{IX})=(\mathrm{VI})^{\prime} .
$$

Since

$$
\mathfrak{R}\left[\left(|f|^{2 \sigma} f\right)^{\prime} \bar{f}\right]=\frac{2 \sigma+1}{2 \sigma+2}\left(|f|^{2 \sigma+2}\right)^{\prime}
$$

we obtain

$$
\begin{aligned}
(\mathrm{X})= & -16 \pi^{3} \Re \sum_{j=1}^{n} \gamma_{j}\left\{\left|q_{j}(t)\right|^{2 \sigma_{j}+2}-\left|q_{j 0}\right|^{2 \sigma_{j}+2}\right. \\
& \left.-\int_{0}^{t} d s q_{j}(s) \frac{d}{d s}\left[\left|q_{j}(s)\right|^{2 \sigma_{j}+2} \overline{q_{j}(s)}\right]\right\} \\
& =(2 \pi)^{3} \sum_{j=1}^{n} \frac{\gamma_{j}}{\sigma_{j}+1}\left[\left|q_{j 0}\right|^{2 \sigma_{j}+2}-\left|q_{j}(t)\right|^{2 \sigma_{j}+2}\right] .
\end{aligned}
$$

From (5.14), (5.29), (5.27), we have

$$
\begin{aligned}
\|\nabla \phi(t)\|_{L^{2}\left(\mathbb{R}^{3}\right)}^{2}= & \left\|\nabla \phi_{0}\right\|_{L^{2}\left(\mathbb{R}^{3}\right)}^{2}+\sum_{j=1}^{n} \frac{\gamma_{j}}{\sigma_{j}+1}\left[\left|q_{j 0}\right|^{2 \sigma_{j}+2}-\left|q_{j}(t)\right|^{2 \sigma_{j}+2}\right] \\
& +\frac{1}{2 \pi} \sum_{j=1}^{n} \sum_{l \neq j} \frac{1}{\left|\mathbf{y}_{j}-\mathbf{y}_{l}\right|}\left\{\Re\left[q_{j}(t) \overline{q_{l}(t)}-q_{j 0} \overline{q_{l 0}}\right]\right\},
\end{aligned}
$$

hence conservation of energy is proven. 


\section{Global existence}

In this section we show global existence of the solution for any initial datum in the case of repulsive or weakly attractive interactions, i.e. for $\gamma_{j}>0$ or $\gamma_{j}<0$ and $\sigma_{j}<1$, $j=1, \ldots, n$. The result is proven in the following theorem:

THEOREM 12. - If for any index $j$ for which $\gamma_{j}<0$ one has $\sigma_{j}<1$ then the solution is global in time for any initial datum $\psi_{0} \in V$.

Proof. - We decompose the initial datum into a regular and a singular part:

$$
\psi_{0}=\phi_{0}+\sum_{k=1}^{n} q_{0 k} G_{0}\left(\cdot-\mathbf{y}_{k}\right), \quad \phi_{0} \in H_{\mathrm{loc}}^{1}\left(\mathbb{R}^{3}\right), \nabla \phi_{0} \in L^{2}\left(\mathbb{R}^{3}\right) .
$$

We know from Theorem 6 that the same decomposition holds for the solution $\psi(t)$ at any time $t$ in the maximal existence interval $\left[0, T^{*}\right)$, namely

$$
\psi(t)=\phi(t)+\sum_{k=1}^{n} q_{k}(t) G_{0}\left(\cdot-\mathbf{y}_{k}\right), \quad \phi(t) \in H_{\mathrm{loc}}^{1}\left(\mathbb{R}^{3}\right), \nabla \phi(t) \in L^{2}\left(\mathbb{R}^{3}\right), t \in\left[0, T^{*}\right) .
$$

For any $t \in\left[0, T^{*}\right)$, let us define the functions $\xi_{j}(t): \mathbb{R} \longrightarrow \mathbb{C}, j=1, \ldots, n$

$$
\xi_{j}(t, x)=|x|\left[T_{j}^{-1} \psi(t)\right]^{m}(|x|), \quad x \in \mathbb{R},
$$

(see definitions (2.4) and (2.2)). One easily sees that

$$
\begin{aligned}
\int_{\mathbb{R}} d x\left|\xi_{j}(t, x)\right|^{2} & =2 \int_{0}^{+\infty} d r r^{2}\left|\left[T_{j}^{-1} \psi(t)\right]^{m}(r)\right|^{2} \\
& \leqslant \frac{1}{2 \pi}\left\|T_{j}^{-1} \psi(t)\right\|_{L^{2}\left(\mathbb{R}^{3}\right)}^{2}=\frac{1}{2 \pi}\left\|\psi_{0}\right\|_{L^{2}\left(\mathbb{R}^{3}\right)}^{2} .
\end{aligned}
$$

Moreover, denoting by $\xi_{j}^{\prime}(t, x)$ the derivative of $\xi_{j}(t, x)$ with respect to $x$, we have

$$
\begin{aligned}
\int_{\mathbb{R}} d x\left|\xi_{j}^{\prime}(t, x)\right|^{2}= & 2 \int_{0}^{+\infty} d r\left|\frac{d}{d r}\left\{r\left[T_{j}^{-1} \phi(t)\right]^{m}(r)+r \sum_{k \neq j} q_{k}(t)\left[T_{j}^{-1} T_{k} G_{0}\right]^{m}(r)\right\}\right|^{2} \\
\leqslant & 4 \int_{0}^{\infty} d r\left|\frac{d}{d r} r\left[T_{j}^{-1} \phi(t)\right]^{m}(r)\right|^{2} \\
& +4(n-1) \sum_{k \neq j}\left|q_{k}(t)\right|^{2} \int_{0}^{\infty} d r\left|\frac{d}{d r} r\left[T_{j}^{-1} T_{k} G_{0}\right]^{m}(r)\right|^{2}
\end{aligned}
$$

We observe that, for any function $F \in H^{1}\left(\mathbb{R}^{3}\right)$ and depending only on the radial coordinate $r$, the following equality holds

$$
\int_{0}^{\infty} d r\left|\frac{d}{d r} r F(r)\right|^{2}=\frac{1}{4 \pi}\|\nabla F\|_{L^{2}\left(\mathbb{R}^{3}\right)}^{2} .
$$


Let us consider the first term in the r.h.s. of (6.5). Using equality (6.6) we find

$$
\int_{0}^{\infty} d r\left|\frac{d}{d r} r\left[T_{j}^{-1} \phi(t)\right]^{m}(r)\right|^{2} \leqslant \frac{1}{4 \pi}\|\nabla \phi(t)\|_{L^{2}\left(\mathbb{R}^{3}\right)}^{2} .
$$

For the second term in (6.5) an explicit computation gives

$$
\left[T_{j}^{-1} T_{k} G_{0}\right]^{m}(r)=\frac{1}{4 \pi\left|\mathbf{y}_{j}-\mathbf{y}_{k}\right|} \chi_{j k}(r)+\frac{1}{4 \pi r}\left(1-\chi_{j k}(r)\right),
$$

where $\chi_{j k}$ is the characteristic function of the interval $\left[0,\left|\mathbf{y}_{j}-\mathbf{y}_{k}\right|\right]$. Using again equality (6.6) we easily get

$$
\int_{0}^{\infty} d r\left|\frac{d}{d r} r\left[T_{j}^{-1} T_{k} G_{0}\right]^{m}(r)\right|^{2}=\frac{1}{16 \pi^{2}\left|\mathbf{y}_{j}-\mathbf{y}_{k}\right|} .
$$

From (6.5), (6.7), (6.9) we conclude

$$
\left\|\xi_{j}^{\prime}(t)\right\|_{L^{2}(\mathbb{R})}^{2} \leqslant \frac{1}{\pi}\|\nabla \phi(t)\|_{L^{2}\left(\mathbb{R}^{3}\right)}^{2}+\frac{n-1}{4 \pi^{2}} \sum_{k \neq j} \frac{\left|q_{k}(t)\right|^{2}}{\left|\mathbf{y}_{j}-\mathbf{y}_{k}\right|}
$$

which means $\xi_{j}(t) \in H^{1}(\mathbb{R})$.

Notice that a straightforward computation in Fourier space gives

$$
\left|r\left[T_{j}^{-1} \phi(t)\right]^{m}(r)\right| \leqslant C r^{1 / 2}\|\nabla \phi(t)\|_{L^{2}\left(\mathbb{R}^{3}\right)}
$$

and then from the definition (6.3) we get

$$
q_{j}(t)=4 \pi \xi_{j}(t, 0) .
$$

From the Sobolev inequality and estimates (6.4), (6.10) we derive the following key estimate of the functions $q_{j}$

$$
\begin{aligned}
\sum_{j=1}^{n}\left|q_{j}(t)\right|^{4} & \leqslant(4 \pi)^{4} \sum_{j=1}^{n}\left\|\xi_{j}(t)\right\|_{L^{2}(\mathbb{R})}^{2}\left\|\xi_{j}^{\prime}(t)\right\|_{L^{2}(\mathbb{R})}^{2} \\
& \leqslant 128 \pi^{3}\left\|\psi_{0}\right\|_{L^{2}\left(\mathbb{R}^{3}\right)}^{2}\left[\frac{n}{\pi}\|\nabla \phi(t)\|_{L^{2}\left(\mathbb{R}^{3}\right)}^{2}+\frac{n-1}{4 \pi^{2}} \sum_{j=1}^{n} \sum_{k \neq j} \frac{\left|q_{k}(t)\right|^{2}}{\left|\mathbf{y}_{j}-\mathbf{y}_{k}\right|}\right] .
\end{aligned}
$$

Substituting the estimate (6.13) into the conservation law of the energy we find

$$
\begin{aligned}
E\left(\psi_{0}\right) \geqslant & C_{1} \sum_{j=1}^{n}\left|q_{j}(t)\right|^{4}+\sum_{\gamma_{k}>0} \frac{\gamma_{k}}{\sigma_{k}+1}\left|q_{k}(t)\right|^{2 \sigma_{k}+2}-\sum_{\gamma_{k}<0} \frac{\left|\gamma_{k}\right|}{\sigma_{k}+1}\left|q_{k}(t)\right|^{2 \sigma_{k}+2} \\
& -\frac{1}{2 \pi} \sum_{l<k} \mathfrak{R} \frac{q_{l}(t) \overline{q_{k}(t)}}{\left|\mathbf{y}_{l}-\mathbf{y}_{k}\right|}-C_{2} \sum_{j=1}^{n} \sum_{k \neq j} \frac{\left|q_{k}(t)\right|^{2}}{\left|\mathbf{y}_{j}-\mathbf{y}_{k}\right|},
\end{aligned}
$$


where $C_{1}, C_{2}$ are positive and time-independent constants.

If we assume that, for some index $j$, there is $T<\infty$ such that $\limsup _{t \rightarrow T}\left|q_{j}(t)\right|=\infty$ then inequality (6.14) is violated. Hence the solution is global in time and the theorem is proven.

Remark 3. - From conservation of energy and (6.13) it is clear that the solution blows up at time $T<\infty$ if and only if $\limsup _{t \rightarrow T}\|\nabla \phi(t)\|_{L^{2}\left(\mathbb{R}^{3}\right)}=\infty$.

The proof of Theorem 12 cannot be extended to the case in which, for some index $j$, one has $\gamma_{j}<0, \sigma_{j} \geqslant 1$. In fact, under these assumptions, we conjecture that one can find a class of initial conditions for which the corresponding solutions blow up in finite time.

Some more information are available in the simpler case of a nonlinearity concentrated in a single point.

PROPOSITION 13. - In the case $n=1, \sigma_{1}=\sigma=1, \gamma_{1}=\gamma<0$, if $\left\|\psi_{0}\right\|_{L^{2}\left(\mathbb{R}^{3}\right)}<$ $(4 \pi \sqrt{2|\gamma|})^{-1}$, then the solution $\psi(t)$ is global in time.

Proof. - Without loss of generality, we choose $\mathbf{y}_{1}=0$ and define:

$$
\xi(t, x)=|x|[\psi(t)]^{m}(|x|)=|x|[\phi(t)]^{m}(|x|)+\frac{q(t)}{4 \pi}, \quad x \in \mathbb{R}, t \in\left[0, T^{*}\right) .
$$

Following the line of Theorem 12, we obtain

$$
\begin{gathered}
\|\xi(t)\|_{L^{2}(\mathbb{R})}^{2} \leqslant \frac{1}{2 \pi}\left\|\psi_{0}\right\|_{L^{2}\left(\mathbb{R}^{3}\right)}^{2}, \\
\left\|\xi^{\prime}(t)\right\|_{L^{2}(\mathbb{R})}^{2} \leqslant \frac{1}{2 \pi}\|\nabla \phi(t)\|_{L^{2}\left(\mathbb{R}^{3}\right)}^{2}, \\
|q(t)|^{2}=16 \pi^{2}|\xi(t, 0)|^{2} \leqslant 16 \pi^{2}\|\xi(t)\|_{L^{2}(\mathbb{R})}\left\|\xi^{\prime}(t)\right\|_{L^{2}(\mathbb{R})} \\
\leqslant 8 \pi\left\|\psi_{0}\right\|_{L^{2}\left(\mathbb{R}^{3}\right)}\|\nabla \phi(t)\|_{L^{2}\left(\mathbb{R}^{3}\right)} \cdot
\end{gathered}
$$

Then

$$
\begin{aligned}
E\left(\psi_{0}\right) & =\|\nabla \phi(t)\|_{L^{2}\left(\mathbb{R}^{3}\right)}^{2}-\frac{|\gamma|}{2}|q(t)|^{4} \\
& \geqslant\|\nabla \phi(t)\|_{L^{2}\left(\mathbb{R}^{3}\right)}^{2}\left(1-32 \pi^{2}|\gamma|\left\|\psi_{0}\right\|_{L^{2}\left(\mathbb{R}^{3}\right)}^{2}\right) .
\end{aligned}
$$

If $\left\|\psi_{0}\right\|_{L^{2}\left(\mathbb{R}^{3}\right)}^{2}<\left(32 \pi^{2}|\gamma|\right)^{-1}$, the solution cannot blow-up, otherwise we would obtain $E\left(\psi_{0}\right)=+\infty$.

Remark 4. - The estimate in proposition 13 on the $L^{2}$-norm is sharp. In fact one can show that the case $\gamma<0, \sigma=1$ is critical, i.e. the evolution problem is pseudoconformal invariant (see $[3,5,6])$. Exploiting this fact one can exhibit a class of blow-up solutions whose $L^{2}$-norm equals $(4 \pi \sqrt{2|\gamma|})^{-1}$.

Moreover in the super critical case $\gamma<0, \sigma>1$ it is possible to prove that there is blow up at least if the energy is negative.

We plan to give a detailed analysis of the blow up solutions in the case of nonlinearity concentrated in a single point in a forthcoming paper. 


\section{REFERENCES}

[1] R. Adami, A. Teta, A Simple Model of Concentrated Nonlinearity, in: Operator Theory: Advances and Applications, Vol. 108, 1999, 183-189.

[2] R. Adami, A. Teta, A class of nonlinear Schrödinger equation with concentrated nonlinearity, J. Funct. Anal. 180 (2001) 148-175.

[3] R. Adams, Sobolev Spaces, Academic Press, New York, 1975.

[4] S. Albeverio, F. Gesztesy, R. Högh-Krohn, H. Holden, Solvable Models in Quantum Mechanics, Springer-Verlag, New York, 1988.

[5] T. Cazenave, An Introduction to Nonlinear Schrödinger Equation, 2nd Edition, in: Textos de Métodos Matematicos, Vol. 26, IMUFRJ, Rio de Janeiro, 1993.

[6] T. Cazenave, Blow up and Scattering in the Nonlinear Schrödinger Equation, in: Textos de Métodos Matematicos, Vol. 30, IMUFRJ, Rio de Janeiro, 1996.

[7] A. Erdely, et al., Tables of Integral Transform, McGraw-Hill, New York, 1954.

[8] J. Ginibre, G. Velo, On a class of nonlinear Schrödinger equations. I. The Cauchy problem, general case, J. Funct. Anal. 32 (1979) 1-32.

[9] R. Gorenflo, S. Vessella, Abel Integral Equations, Springer-Verlag, Berlin, 1978.

[10] F. Kappel, K. Kunisch, Invariance results for Delay and Volterra equations in fractional order Sobolev spaces, Trans. Math. Soc. 304 (1987)(1), 1-51.

[11] T. Kato, On nonlinear Schrödinger equations, Ann. Inst. Henri Poincaré, Physique Théorique 46 (1987) 113-129.

[12] R.K. Miller, Nonlinear Volterra Integral Equations, W.A. Benjamin, 1971.

[13] M.R. Sayapova, D.R. Yafaev, The evolution operator for time-dependent potentials of zero radius, Proc. Stek. Inst. Math. 2 (1984) 173-180.

[14] A. Teta, Quadratic forms for singular perturbations of the Laplacian, Publ. R.I.M.S. Kyoto Univ. 26 (1990) 803-817.

[15] M.I. Weinstein, NLSE and sharp interpolation estimates, Comm. Math. Phys. 87 (1982/83) $567-576$. 